\section{KENTRON}

REVUE PLURIDISCIPLINAIR

DU MONDE ANTIQUE

\section{Kentron}

Revue pluridisciplinaire du monde antique

$25 \mid 2009$

Histoire et actualité des sciences de l'Antiquité

\title{
La philologie classique et l'apprentissage de la lecture intelligente
}

\section{Laurent Calvié}

\section{OpenEdition}

1 Journals

\section{Édition électronique}

URL : http://journals.openedition.org/kentron/1461

DOI : 10.4000/kentron.1461

ISSN : 2264-1459

\section{Éditeur}

Presses universitaires de Caen

\section{Édition imprimée}

Date de publication : 31 décembre 2009

Pagination : 29-52

ISBN : 978-2-84133-343-1

ISSN : 0765-0590

\section{Référence électronique}

Laurent Calvié, «La philologie classique et l'apprentissage de la lecture intelligente », Kentron [En ligne], 25 | 2009, mis en ligne le 12 mars 2018, consulté le 19 novembre 2020. URL : http:// journals.openedition.org/kentron/1461; DOI : https://doi.org/10.4000/kentron.1461

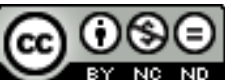

Kentron is licensed under a Creative Commons Attribution-NonCommercial-NoDerivatives 3.0 International License. 


\section{LA PHILOLOGIE CLASSIQUE ET L'APPRENTISSAGE DE LA LECTURE INTELLIGENTE}

La philologie, ça rend les gens intelligents.

Henri-Irénée Marrou ${ }^{1}$

Les premières éditions des Euvres posthumes d'André Chénier (1762-1794) sont unanimes. Au début d'un de ses Iambes, le jeune et génial poète aurait écrit:

Quand au mouton bêlant la sombre boucherie

Ouvre ses cavernes de mort,

Pauvres chiens et moutons, toute la bergerie

Ne s'informe plus de son sort ${ }^{2}$.

En 1861, cependant, un professeur de l'université de Besançon affirma, dans le Journal général de l'instruction publique, que le troisième vers de cette strophe était corrompu et qu'il fallait lire:

Quand au mouton bêlant la sombre boucherie

Ouvre ses cavernes de mort,

Pâtres, chiens et moutons, toute la bergerie

Ne s'informe plus de son sort ${ }^{3}$.

J'ignore si l'«hémistiche d'André Chénier lui doit son salut», comme l'a affirmé Théodore Reinach (1860-1928) ${ }^{4}$, puisque cette restitution a été confirmée depuis par le manuscrit original ${ }^{5}$; mais je sais qu'une telle correction, qu'on appelle correction palmaire parce qu'elle paraît évidente à tous une fois faite ${ }^{6}$, n'était nullement à la portée du premier venu.

1. Remarque orale rapportée dans Vidal-Naquet 2006, 46.

2. Cf. Chénier 1819, 268; Chénier 1826, 308; Chénier 1832, 139; et Chénier 1852, 273.

3. Cf. Weil 1861 a, 279.

4. Cf. Reinach 1909, 381 .

5. Cf. Chénier 1874, 283 et 366 : «Le premier éditeur a lu ainsi ce vers: Pauvres chiens et moutons, toute la bergerie. L'erreur a été reproduite par les éditions subséquentes et par l'édition critique de $1862 »$; voir aussi Chénier 1958, 192.

6. Cf. Carcopino 1968, VIII: «Correction palmaire est une locution courante dans la langue des philologues. Mais, spéciale à leur milieu, elle n'a pas acquis droit de cité dans les meilleurs des 


\section{Les fruits de l'enseignement philologique}

Le français n'était pourtant pas la langue maternelle de son inventeur, puisque celui-ci «était né en 1818, à Francfort-sur-le-Mein » et n'avait été naturalisé qu'en $1848^{7}$. Henri Weil (1818-1909), puisque c'est de lui qu'il s'agit, n'avait pas non plus fait d'études supérieures de langue ou de littérature française: en 1838, il avait été reçu Docteur en philosophie summa cum laude de l'université de Leipzig, après avoir brillamment soutenu une thèse sur l'écriture de l'histoire chez Thucydide ${ }^{8}$; et, en 1845 , Docteur ès lettres de l'université de Paris, après avoir non moins brillamment soutenu une thèse latine sur les allusions historiques dans la tragédie grecque ${ }^{9}$ et une thèse française sur l'ordre des mots dans les langues classiques ${ }^{10}$. Il n'était cependant à proprement parler ni philosophe, ni historien, ni linguiste, ni même littéraire. Bien que Georges Perrot (1832-1914) ait fait l'éloge de sa maîtrise de la langue française et de son style ${ }^{11}$, Henri Weil ne sut jamais bien les finesses de notre idiome national et de sa phraséologie, qu'il teinta toujours d'une charmante touche d'exotisme germanique. Anatole France (1844-1924), qui a peint le vieux Weil sous les traits de «M. Schmoll, de l'Académie des inscriptions ", dans Le lys rouge $(1894)^{12}$, l'a souligné à juste titre, dans un passage de ce roman qui se ressent clairement de l'antisémitisme du temps:

Il souriait, grimaçant et poupin; il tournait des madrigaux à la comtesse Martin avec cette voix héréditaire, rude et grasse, dont les Juifs ses pères pressaient leurs débiteurs, les paysans d'Alsace, de Pologne et de Crimée. Il traînait lourdement ses phrases. Ce grand philologue, membre de l'Institut de France, savait toutes les langues, excepté le français. Et madame Martin s'amusait de ces galanteries lourdes et rouillées comme les ferrailles qu'étalent les brocanteurs, et parmi lesquelles tombaient quelques fleurs séchées de l'Anthologie ${ }^{13}$.

dictionnaires modernes, tels que le Grand Larousse encyclopédique ou le Robert. Elle signifie la récompense qui vient, avec les palmes d'une victoire, couronner l'érudit assez heureux pour avoir redressé la faute d'un texte par un changement de terme, de syllabe ou, simplement, de lettre, d'une si aveuglante évidence qu'il semble inutile de le justifier par une démonstration en règle».

7. Cf. Perrot 1910, 5 et 31. Je profite de l'occasion pour signaler que la notice de Perrot sera bientôt rééditée par mes soins dans Weil 2010.

8. Cf. Weil 1838.

9. Cf. Weil 1844 a.

10. Cf. Weil 1844 b.

11. Cf. Perrot 1910, 5: «Quoiqu'il écrivît le français avec une propriété de termes et même avec une élégance que pouvaient lui envier beaucoup même de ceux qui, parmi nos compatriotes, font métier d'écrivain, il ne nous appartenait que par l'adoption. C'était l'Allemagne qui nous l'avait donné».

12. Cf. Hemmerdinger 1972, 96: «Elle [sc. l'aînée des petites filles d'Henri Weil, $\mathrm{M}^{\mathrm{me}}$ Dufour] m’apprend qu'Anatole France a connu Henri Weil au cours d'une excursion dans le Hohwald. Le Lys rouge est un roman à clef où Henri Weil figure sous le nom de Joseph Schmoll».

13. Cf. France 1924, 13. 
À première vue, ce n'était donc pas vraiment le candidat idéal à la restitution d'un vers corrompu d'un poète classique français que cet ancien élève du comparatiste Franz Bopp (1791-1867) et de Friedrich Gottlieb Welcker (1784-1868), August Bockh (1785-1867) et Gottfried Hermann (1772-1848), tous trois passés maîtres en cette science que Friedrich August Wolf (1759-1824) venait de baptiser AltertumsWissenschaft (science de l'Antiquité) et avait ainsi définie, dans sa Darstellung der Altertums-Wissenschaft, publiée à Berlin en 1807:

La science de l'Antiquité comprend l'ensemble des études qui nous font connaître les actions et les destinées des Grecs et des Romains, leur état politique, intellectuel et domestique, leur civilisation, leurs langues, leurs arts et leurs sciences, leurs mœurs et leurs religions, leurs caractères nationaux et leurs idées, afin de nous mettre à même de comprendre parfaitement leurs œuvres et de nous en approprier la substance et l'esprit, en évoquant la vie antique et en la comparant à celle des âges suivants et à la nôtre ${ }^{14}$.

Henri Weil pouvait ainsi avoir des compétences certaines dans des domaines aussi divers que ceux de l'histoire ancienne, de la stylistique grecque, de la grammaire comparée des langues classiques et de l'histoire de la littérature gréco-romaine, puisque c'était là autant de domaines impartis à la science de l'Antiquité. Mais cela ne le prédestinait guère à corriger le texte corrompu d'un des Iambes d'André Chénier, là où les éditeurs autochtones n'avaient pas même subodoré la moindre corruption. S'il en a été capable, ce n'est sans doute pas parce qu'il était devenu un spécialiste de l'Alterthumswissenschaft, mais parce qu'il avait reçu de ses maîtres allemands, August Bockh ${ }^{15}$ et Gottfried Hermann ${ }^{16}$, un enseignement philologique

14. Cf. Wolf 1807,30 (traduit dans Bonnet 1892, 66).

15. Cf. Perrot 1910, 15 sq.: «L'éducation du jeune helléniste était ainsi bien commencée. Au bout d'un an, ce fut à Berlin qu'elle alla s'achever [...]. C'est qu'il avait été d'emblée pris tout entier et comme conquis par l'enseignement de Boeckh. Ce qui l'avait charmé tout d'abord, c'était l'admirable clarté de la parole du maître, soit que celui-ci exposât la méthode et le système de la philologie, telle qu'il la comprenait, soit qu'il étudiât les institutions des cités de la Grèce, soit qu'il expliquât un dialogue de Platon. Weil entra donc dans le séminaire de Boeckh, où, de jour en jour, il apprécia davantage la supériorité de cette puissante intelligence. Avec sa force prodigieuse de travail, Boeckh avait tout appris de l'antiquité grecque. Il en savait tout; mais ces études diverses, ces recherches spéciales dont chacune paraissait à d'autres savants se suffire à elle-même et mériter d'occuper, à elle seule, toute une existence, il les faisait toutes converger vers un but suprême qu'il ne perdait jamais de vue. Chacune d'elles n'était pour lui qu'un moyen, le moyen de saisir et de fixer quelques-uns des traits dont le rapprochement donnerait une image d'ensemble, celle de la vie publique et privée des sociétés grecques. C'était avec cette préoccupation qu'il lisait et qu'il enseignait à lire les auteurs classiques [...] ».

16. Ibid., 18: «Pour terminer ses études, il lui fallut donc se transporter encore dans une autre Université. Ce fut Leipzig qu'il choisit. Il y était attiré par la renommée du célèbre helléniste Gottfried Hermann. Les cours de Gottfried Hermann intéressèrent l'étudiant; mais celui-ci ne voua point à ce nouveau maître les sentiments de vive sympathie que la personne même de Boeckh lui avait tout d'abord inspirés [...]». 
d'une excellente qualité, dont on n'a pas toujours bien vu l'unité fondamentale. Depuis Wilamowitz ${ }^{17}$, on a ainsi beaucoup exagéré l'importance du différend qui opposa ces deux maîtres incontestés de la philologie allemande d'alors sur la nature et la fin de leur discipline ${ }^{18}$ : l'un et l'autre plaçaient en effet au centre de celle-ci la lecture des textes anciens et pratiquaient allègrement la critique conjecturale (Konjekturalkritik) ${ }^{19}$; et c'est probablement leurs leçons qui mirent Henri Weil en état de corriger le texte corrompu d'André Chénier:

Hermann était et voulait rester étranger à cette large conception historique de l'antiquité qui était sans cesse présente à la pensée de Boeckh et de son génial ami Karl Otfried Müller. Tout ce qu'il avait de science et d'esprit, il l'appliquait à la critique des textes; il l'employait à discuter les leçons des manuscrits, à tâcher d'en combler les lacunes et d'en corriger les fautes par d'ingénieuses conjectures. Boeckh était loin de considérer ce travail comme inutile et il rendait un juste hommage à ceux qui, comme Hermann, y excellaient. Comme éditeur de textes, Boeckh avait fait ses preuves par son édition de Pindare. Il les fit mieux encore par les soins qu'il donna, lors de la publication du Corpus, à l'établissement du texte de tant d'inscriptions qui avaient été si souvent mal transcrites et où manquaient partout des mots et des phrases entières; mais ce qu'il avait peine à comprendre, c'était qu'un esprit de haute valeur se cantonnât dans cette étude sans essayer jamais de mettre à profit les résultats ainsi obtenus pour renouveler, en l'éclairant d'un meilleur jour, l'un des aspects sous lesquels s'offre à notre curiosité ce monde grec dont rien ne saurait nous être indifférent. De l'une à l'autre des deux tendances, le désaccord était trop marqué pour ne pas donner lieu à quelques polémiques entre les deux chefs d'école. Ceux-ci commencèrent par se combattre à armes courtoises; puis quelque aigreur finit par se mêler au débat.

Tout en admirant la virtuosité d'Hermann, Weil était resté, de cœur et de pensée, sous l'influence du maitre de Berlin. Lui qui devait plus tard se signaler, surtout comme éditeur d'Eschyle, par les libertés qu'il prendrait avec les leçons des manuscrits, il se tenait sur la réserve, au temps où il suivait les cours d'Hermann. Les corrections et les conjectures que celui-ci proposait à ses auditeurs lui faisaient un peu l'effet de jeux d'esprit et d'amusements frivoles. Tout mal disposé qu'il fût ainsi pour le rival et le contradicteur du maître auquel il était attaché, il ne put manquer de tirer plus de profit qu'il ne croyait alors de l'enseignement d'un helléniste aussi savant que l'était Hermann ${ }^{20}$.

17. Cf. Wilamowitz-Moellendorf 1921, 242-244;

18. Cf. Bøckh 1991, 8-14.

19. Weil voit juste quand il ramène la dissension entre les deux philologues à une question de degré (cf. Weil 189o, 213): «Signalons quelques pages qu'on lira avec intérêt, celles où il [Wilamowitz] raconte la querelle qui divisa naguère les deux écoles rivales, l'une plus occupée des textes littéraires, l'autre plus ouverte aux études historiques, et représentées, la première par Gottfried Hermann, la seconde par August Bœckh et Otfried Müller» [c'est moi qui souligne].

20. Cf. Perrot 1910, 19. 
Jean Bollack a déjà fait remarquer la tendance marquée d'Henri Weil à la pratique de la critique conjecturale:

Des deux orientations majeures de la philologie allemande de ce temps, l'analyse des œuvres, en premier lieu des poèmes homériques, à savoir leur découpage et recomposition, et l'exercice conjectural, les applications et variations étaient innombrables [...]. Henri Weil avait opté pour la seconde, par goût, et sans doute par instinct, sentant que la seconde était plus sérieuse, et que la première allait contre le bon sens et choquerait en France ${ }^{21}$.

Rien ne témoigne cependant mieux de son goût pour cet exercice éminemment philologique qu'une série de petits mémoires qu'il donna vers 1860 au Journal général de l'instruction publique et à la Revue de l'instruction publique ${ }^{22}$, dont l'un a tout bonnement pour titre "De la méthode à suivre pour restituer les textes anciens ${ }^{23}$ et dont un autre offre précisément la restitution du vers de Chénier mentionnée en tête du présent article, qu'il accompagne de l'observation suivante:

Depuis la découverte de l'imprimerie, la pureté des textes est infiniment mieux garantie; et cependant la presse elle-même laisse quelquefois échapper des fautes qui se propagent ensuite: les publications posthumes surtout sont exposées à des erreurs presque inévitables. Certes, les poésies d'André Chénier ont été recueillies avec un grand soin et un pieux dévouement. Mais croit-on que le poète ait pu écrire ce qu'on lit dans l'iambe où il se plaint que ses amis l'oublient depuis qu'il est en prison: [...]. Pauvres chiens. Je ne sais si le chien du berger est à plaindre, mais ici l'épithète d'indifférent, d'insensible lui conviendrait mieux que celle de pauvre. Quiconque est un peu versé dans la critique des textes grecs et latins vous dira de suite qu'il y a ici une faute, et vous indiquera la correction à faire. Dans la bergerie, il n'y a pas seulement des chiens et des moutons, on ne peut y oublier le berger. Chénier a dû écrire: «Pâtres, chiens et moutons, toute la bergerie $[\ldots]{ }^{24}$.

J'ai dit plus haut qu'on avait beaucoup exagéré l'importance du différend qui opposa les deux maîtres incontestés de la philologie allemande d'alors sur la nature et la fin de leur discipline. Tout comme Gottfried Hermann, August Bœekh, qu'Henri Weil appelait en 1855 son "vénéré maître et le maître de tous ceux qui étudient l'Antiquité» ${ }^{25}$, n'a en effet jamais admis le néologisme (die Altertumswissenschaft) proposé par Wolf pour désigner ce qu'il appelait pour sa part la philologie ou la

21. Cf. Bollack 1996, 34 .

22. Cf. Weil 1847; Weil 1859 ; Weil 1861 a; Weil 1861 b et Weil 1866.

23. Cf. Weil 1866. Cet article a échappé à l'attention des rédacteurs de sa seule bibliographie (Glotz \& Reinach 1909); je ne l'ai trouvé mentionné que dans Peyre 1932, 198.

24. Cf. Weil 1861, 279.

25. Cf. Weil \& Benloew 1855, V (dédicace). 
"science philologique» (die Philologie, die philologische Wissenschaft), et il a d'emblée réclamé contre la conception wolfienne de la science de l'Antiquité, qu'il jugeait par trop entachée d'empirisme. Sa posthume Encyklopädie und Methodologie der philologischen Wissenschaften (1877), dont les linéaments remontent à $1807^{26}$, s'ouvre ainsi sur la critique suivante (I, $\$ 1$ ):

Le concept d'une science ou d'une discipline scientifique n'est pas donné par une énumération discontinue des éléments contenus en elle [...]. Le véritable concept de toute science, et donc aussi de la philologie, si tant est qu'elle doive comporter une part de science, doit être tel à l'égard des parties de celle-ci, que l'ensemble du concept en comprenne toutes les parties, que les parties en soient toutes contenues en lui comme concepts et que chaque partie en présente à son tour le concept tout entier. La définition de la philologie qui procède par l'énumération de ses parties ne vaut guère mieux que la définition du beau que Platon met dans la bouche d'Hippias, dans l'Hippias majeur: «Le beau est une belle jeune femme, de l'or, etc. ${ }^{27}$.

Selon lui, la philologie ne pouvait pas davantage se réduire à l'étude de l'Antiquité qu'à celle du langage, ou encore à l'érudition, à la critique, à l'histoire de la littérature ou aux humanités: «la tâche qui incombait en propre à la philologie devait résider dans la connaissance des productions de l'esprit humain, c'est-à-dire du connu» ${ }^{28}$. L'associant et l'opposant tout à la fois à la philosophie, il écrivait ainsi $\left(\mathrm{I}, \S_{3}\right)$ :

La philologie ayant pour but de reconnaître et d'interpréter tout le savoir humain dont elle dispose, elle est associée à la philosophie, au point qu'elle plonge ses racines dans cette dernière, dans la connaissance de l'esprit; et elle ne se différencie de la philosophie que par le mode de connaissance qui est le sien: la philosophie connaît primitivement, $\gamma \iota \gamma v \dot{\omega} \sigma \kappa \varepsilon \mathrm{l}$, la philologie connaît secondairement [connaît

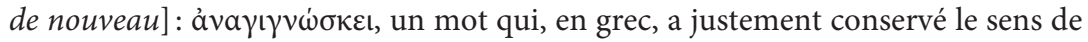
«lire», puisque la lecture est une activité éminemment philologique, l'instinct de lire étant la première expression de l'instinct philologique ${ }^{29}$.

Ce faisant, August Bœckh, comme Gottfried Hermann avant lui ${ }^{30}$, inscrivait la toute moderne philologie classique dans l'ancienne tradition philologique inaugurée

26. Cf. Bœeckh 1991, 28.

27. Cf. Bœeckh 1877, 5 .

28. Ibid., 10.

29. Ibid., 16 .

30. Cf. Wilamowitz-Moellendorf 1921, 239: «Die hermannische Philologie ist noch durchaus die antike, oder vielmehr wieder die antike, denn er schließt sich weder bewußt noch durch Tradition an die Definition des Dionysios Thrax», que je traduirais ainsi: «La philologie de Hermann est en tout point la philologie antique ou, plutôt, elle est de nouveau la philologie antique, car elle ne se rattache ni consciemment ni par tradition à la définition de Denys le Thrace». 
par les grammairiens alexandrins, qui définissaient leur art comme la pratique de la lecture experte des textes littéraires:

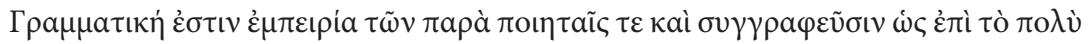

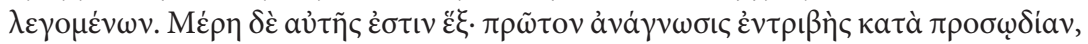

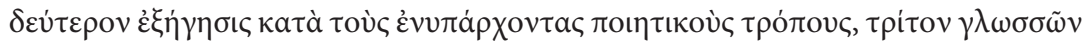

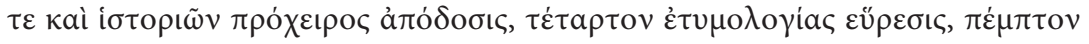

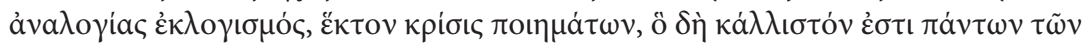
$\dot{\varepsilon} \nu \tau \tilde{\eta} \tau \dot{\varepsilon} \chi \vee \eta^{31}$.

Qu'on ne s'y trompe pas: ce qu'à la suite du grammairien Antidore de Cumes ${ }^{32}$, Denys le Thrace nommait $\gamma \rho \alpha \mu \mu \alpha \tau$ ı́ (la grammaire, ou mieux, l'étude des lettres) correspond bien à ce que nous appelons ici la philologie. Suétone suit le même usage, lorsque, dans son De grammaticis, il présente la grammatica comme la spécialité de Cratès de Mallos et d'Aristarque:

Primus igitur, quantum opinamur, studium grammaticae in urbem intulit Crates Mallotes, Aristarchi aequalis, [...] missus ad senatum ab Attalo rege inter secundum ac tertium Punicum bellum sub ipsam Enni mortem ${ }^{33}$.

Cratès de Mallos ne fut pas, en effet, un grammairien dans l'acception moderne du terme, mais bien un philologue, comme l'indiquent les mentions de son nom dans les scholies d'Hésiode, d'Euripide, d'Aratos et d'Apollonios de Rhodes, les titres conservés de certains de ses ouvrages (Amendements de l'Iliade et de l'Odyssée, Études homériques ou bien encore Sur le dialecte attique), ainsi que les traces de ses recherches étymologiques et de ses catalogues d'auteurs de la bibliothèque de

31. Denys le Thrace (Lallot 1989), 1, 41: «La grammaire est la connaissance empirique de ce qui se dit couramment chez les poètes et les prosateurs. Elle a six parties: premièrement, la lecture experte respectueuse des diacritiques; deuxièmement, l'explication des tropes poétiques présents (dans le texte); troisièmement, la prompte élucidation des mots rares et des récits; quatrièmement, la découverte de l'étymologie; cinquièmement, l'établissement de l'analogie; sixièmement, la critique des poèmes - qui est, de toutes les parties de l'art, la plus belle». Sur les débats antiques autour de cette définition traditionnelle de la grammaire, on lira avec intérêt la revue critique de Sextus Empiricus dans son Contre les grammairiens (57-90), traduite en français dans Baratin \& Desbordes 1981, 142-149. Sur la branche latine de cette tradition (Varron, Suétone, Diomède, Marius Victorinus), voir Dahlmann 1970, 8-12.

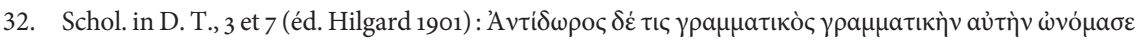

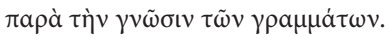

33. Cf. gramm. 2, 1, que je traduirais ainsi: «Le premier, donc, si je ne me trompe, qui introduisit à Rome les études philologiques fut Cratès de Mallos, un contemporain d'Aristarque: il avait été envoyé auprès du sénat par le roi Attale, entre la deuxième et la troisième guerre punique, vers l'époque de la mort d'Ennius»; voir aussi Quintilien, inst. 2, 1, 4. 



diqua le premier», suivant le même Suétone ${ }^{35}$, paraît avoir été utilisé dès l'Antiquité comme un terme générique, sous lequel pouvaient être subsumés ceux de grammairien ( $\gamma \rho \alpha \mu \mu \alpha \tau \kappa o ́ \varsigma$, grammaticus), entendu en un sens restreint, et de critique (крıтıкóৎ, criticus) - deux termes que les scholiastes anciens de Denys le Thrace

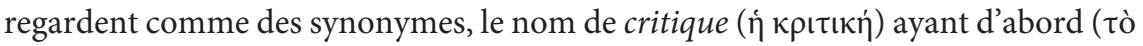
$\pi \rho o ́ \tau \varepsilon \rho o v)$ servi, par synecdoque de la partie pour le tout, à désigner l'ensemble de la grammaire ( $\dot{\eta} \gamma \rho \alpha \mu \mu \alpha \tau \kappa \kappa \dot{)}$ ), parce que c'était le nom de «la plus belle de toutes

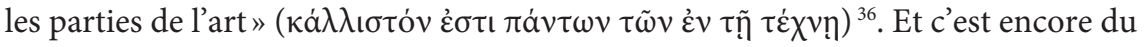
nom de critique (critica) que se servait Henri de Valois (1603-1676), dans les deux livres de son De critica, pour désigner la philologie, telle qu'elle fut conçue en Allemagne, dans la première moitié du XIX ${ }^{e}$ siècle:

Superiore Libro de iis qui apud veteres Critici sunt dicti \& de Criticae praestantia abunde disserui. Ex quibus apparet longe opinione falli eos qui hanc artem tanquam vilem \& abjectam, non solum fastidio, sed etiam contemtu dignam esse arbitrantur. Nunc quid sit \& quot quasque in partes dividatur accuratius discendum est. Ac primum Criticam ita definio, artem judicandi de scriptis tam veterum quam recentiorum. Hic enim praecipue scopus est hujus artis, ut tam de Poematis quam de Orationibus, Historiis, Dialogis, omni denique genere scriptionis recte judicare valeamus. Partes ejus quatuor sunt: prima est expositio seu enarratio, quae Graece

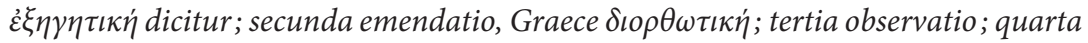
judicium seu censura ${ }^{37}$.

Je crois donc que, si Henri Weil fut capable de corriger un vers de Chénier dont la corruption effective avait échappé à ses premiers éditeurs, ce n'est pas seulement que l'enseignement philologique à lui dispensé par August Bœckh et

34. Sur les ouvrages philologiques de Cratès de Mallos, voir Flamand 1994, 490 sq.

35. Cf. gramm. 10, 4: «Eratosthenes [...] primus hoc cognomen [philologi appellationem] sibi uindicauit».

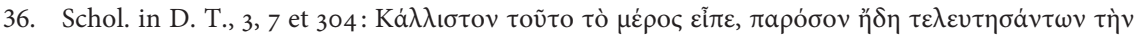

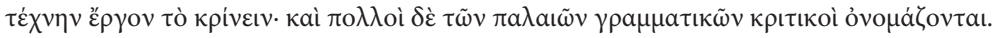

37. Cf. Valois 1740, 184, que je traduirais ainsi: «Dans le livre précédent, j’ai abondamment parlé de ceux que les Anciens appelaient les critiques et de la haute estime dans laquelle ils tenaient la critique. C'est donc une lourde erreur que de la considérer comme une matière vile et abjecte, non seulement fastidieuse, mais encore méprisable. Il faut maintenant dire plus précisément ce qu'elle est et en combien de parties elle est divisée. Voici donc d'abord la définition que j'en propose : la critique est l'art de juger des écrits tant anciens que modernes. C'est en effet là l'objectif premier de cet art: nous rendre capables de juger correctement tant des poèmes que des discours, des histoires, des dialogues et, enfin, des écrits de toute sorte. Elle comprend quatre parties: la première est l'explication, ou interprétation

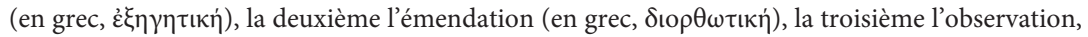
la quatrième le jugement, ou censure». Qu'on me permette ici de signaler la parution prochaine, aux Belles Lettres, du texte latin et d'une traduction française du De critica d'Henri de Valois. 
Gottfried Hermann l'avait rendu habile en l'art de la critique conjecturale, mais c'est qu'il lui avait donné les moyens de pratiquer une lecture experte, c'est-à-dire une lecture intelligente.

\section{Une enseignante de lente lecture}

Il est une pensée détachée de Friedrich Ritschl (1806-1876), qui indique toute la place que doit tenir la lecture dans les études philologiques. Elle appartient à l'esquisse d'un ouvrage auquel songea le grand philologue durant les quinze dernières années de sa vie et dont les bribes ont été publiées sous le titre de «Zur Methode des philologischen Studiums » ${ }^{38}$. La voici : «Lire, lire beaucoup, lire énormément, lire le plus possible $»^{39}$. Il faut cependant se garder de réduire la lecture philologique à son aspect quantitatif. Le plus célèbre disciple de Ritschl, Friedrich Nietzsche (1844-1900), est à cet endroit formel. Il écrit en effet dans l'avant-propos d'Aurore (1881):

En outre nous sommes tous deux des amis du lento, moi et mon livre. On n'a pas été philologue en vain, on l'est peut-être encore, ce qui veut dire professeur de lente lecture [...]. La philologie, effectivement, est cet art vénérable qui exige avant tout de son admirateur une chose: se tenir à l'écart, prendre son temps, devenir silencieux, devenir lent, - comme un art, une connaissance d'orfèvre appliquée au mot, un art qui n'a à exécuter que du travail subtil et précautionneux et n'arrive à rien s'il n'y arrive lento. C'est en cela précisément qu'elle est aujourd'hui plus nécessaire que jamais, c'est par là qu'elle nous attire et nous charme le plus fortement au sein d'un âge de «travail», autrement dit: de hâte, de précipitation indécente et suante qui veut tout de suite «en avoir fini» avec tout, sans excepter l'ensemble des livres anciens et modernes: - quant à elle, elle n'en a pas si aisément fini avec quoi que ce soit, elle enseigne à bien lire, c'est-à-dire lentement, profondément, en regardant prudemment derrière et devant soi, avec des arrière-pensées, avec des portes ouvertes, avec des doigts et des yeux subtils ${ }^{40} \ldots$

38. Sur la conception que Ritschl se faisait de la philologie, voir Benoist $1877,99 s q .:$ «Les vues de M. Ritschl sur la science étaient d'ailleurs hautes et larges. L'idée qu'il se faisait de la philologie était étendue. Il ne la borne point à la critique verbale, à la paléographie, aux recherches grammaticales, à telle ou telle branche isolée de ce qu'elle doit être. C'est pour lui la reproduction de la vie de l'antiquité classique par la connaissance et la considération de ses expressions essentielles. Elle comprend l'étude de l'histoire politique, des religions, de l'art et de l'archéologie, de l'histoire littéraire, en même temps que celle de la grammaire, de l'herméneutique et de la critique. L'application aux textes doit mener à la connaissance des faits et surtout à celles des sentiments et des idées qui ont occupé les âmes et les esprits dans le monde ancien. Il semble qu'il ait attaché une grande importance à propager cette doctrine élevée et féconde. Car, entre les programmes qui contiennent l'indication de ses cours, il en est peu où, à côté des interprétations de textes, on ne voie annoncées des leçons sur la méthode générale et les principes de la philologie».

39. Cf. Ritschl 1879, 28: «Lesen, viel lesen, sehr viel lesen, möglichst viel lesen».

40. Cf. Nietzsche 1989, 18; texte original édité dans Nietzsche 1954, 1016. 
On se peut faire une idée assez claire de la nature de l'apprentissage philologique de cette lente et bonne lecture grâce au témoignage d'un autre disciple de Ritschl, le métricien Wilhelm Brambach (1841-1932), qui a rendu compte de l'enseignement de son maître dans son Friedrich Ritschl und die Philologie in Bonn:

Le centre de toutes les études philologiques réside dans la compréhension exacte et dans l'explication universelle des classiques. Comment un jeune étudiant peut-il donner une explication universelle des auteurs, sans maîtriser toutes les disciplines? Quand le lycéen préparait sa lecture, il s'aidait d'une grammaire et d'un dictionnaire, auxquels il demandait l'explication tant des mots que des principales réalités, pour parvenir à la compréhension. S’il n'y parvient pas, il lui reste, en dernière instance, le professeur, dont il peut prendre l'avis durant les heures de cours. L'étudiant fait de même, à ceci près, qu'il remplace l'autorité du professeur par celle d'un commentateur. Il se peut qu'il ne soit pas satisfait de l'avis de celui-ci: c'est alors un signe qu'il est prêt à se spécialiser et commence à penser par lui-même [...]. Il se renseigne sur la tradition (il apprend à connaître les codices d'un auteur et leur filiation), explique cette tradition, en combinant le sens du texte transmis avec les lois de la pensée logique, celles de la grammaire (métrique), et avec les résultats des recherches historiques et archéologiques (il pratique l'exégèse). S'il ne parvient pas à les combiner, il devra rechercher toutes les voies qu'ont prétendu tracer les commentateurs antérieurs (il s'approprie la bibliographie moderne d'un auteur). Il distingue alors les voies praticables et les impasses (il associe la critique à l'exégèse); et, si toutes ces voies lui paraissent finalement être des impasses, il conclut que la tradition doit être corrompue et cherche à la mettre en accord avec la métrique et ce qu'on appelle les réalités - grammaire comprise -, en l'isolant de ce qui la corrompt (il pratique la critique textuelle) ${ }^{41}$.

Près d'un siècle plus tard, l'apprentissage de la lente lecture se pratiquait encore d'une manière bien peu différente, à Bâle, comme en témoigne Jean Bollack (né en 1923), qui y était étudiant en philologie grecque durant «les années sombres de la guerre»:

Une grande tradition universitaire s'y survivait d'une autre manière à elle-même, protégée et libre, comme dans une réserve. Bâle était restée ce que l'Allemagne avait eu de plus sérieux, de plus irréel et de plus utopique [...].

Dans les séminaires institutionnels que j'ai connus à Bâle, mais surtout dans celui de Von der Mühll ${ }^{42}$, les conditions d'une recherche commune étaient réunies.

41. Cf. Brambach 1865,9 sq.

42. Sur Peter Von der Mühll (1885-1970), cf. Simon-Nahum 2003, 334: «Philologue suisse, lui-même élève au début du siècle de trois grands maîtres des études anciennes: Friedrich Leo (latin), Eduard Schwartz (grec) et Jakob Wackernagel (linguistique indo-européenne) à Göttingen - ce qui faisait de lui, indirectement, le disciple du maître de la discipline Wilamowitz-Moellendorff». 
La transmission des techniques du savoir s'effectuait implicitement par la discussion; le sens des textes restait à trouver. Leur opacité était postulée, réaffirmée secondairement, en dépit de leur fortune scolaire, et s'offrait au travail d'élucidation. Nous restions parfois un quart d'heure, et souvent sans rien dire pendant des minutes entières, sur une phrase que le maître disait sérieusement ne pas comprendre; il en exposait les difficultés, accueillait les propositions, stimulait la réflexion ${ }^{43}$.

À vrai dire, elle se pratique encore de nos jours partout où l'on cherche sérieusement à établir le sens d'un texte, que ce soit à Lille, à Paris ou à Aix-en-Provence; car les bienfaits de la lecture philologique sont reconnus depuis longtemps, y compris en dehors du champ restreint des rares spécialistes de la philologie classique. Un historien de la philosophie comme Jacques Brunschwig écrivait ainsi dans «Faire de l'histoire de la philosophie aujourd'hui» (1976):

Les textes des philosophes grecs, comme tous ceux de l'Antiquité, nous sont parvenus dans des conditions précaires; entre leurs auteurs et nous s'interposent une immense troupe de copistes, de paléographes, des philologues; ce qu'ils nous donnent à comprendre est un objet usé par le temps, indissolublement préservé et détérioré par les intermédiaires matériels et humains qui, en s'employant à le préserver, ont pris en même temps le risque de le détériorer. Ensuite, parce que le grec est pour nous une langue étrangère et une langue morte, et qu'il n'y a aucun intérêt à l'oublier, même si l'on croit habile de le feindre pour en sauvegarder la place dans notre enseignement: en fait, l'essentiel de son charme et de son intérêt tient peut-être à ce qu'il ne délivre aucune signification qui ne doive être conquise par un travail méthodique, conscient de soi, et sujet à l'erreur [...]. C'est un travail faillible que de déterminer quelles ont été les «choses dites" par un philosophe; c'est donc un travail qui engage une responsabilité, et qui, comme tel, vaut la peine que l'on essaie de le faire correctement et techniquement ${ }^{44}$.

C'est même chez un autre historien de la philosophie contemporain, Rémi Brague, qu'on trouve la meilleure description de la méthode philologique de la lecture intelligente. Dans la conclusion de son Du temps chez Platon et Aristote (1982), il affirme en effet que celle-ci « est plus féconde que l'ensemble des résultats auxquels elle peut mener» et ajoute:

À chaque fois, on l'aura remarqué aisément, et non peut-être sans quelque agacement, le point de départ choisi est un détail assez ténu portant sur la lettre du texte: quelle leçon faut-il choisir lorsque les manuscrits ne paraissent pas pouvoir satisfaire, et quand aucune correction déjà proposée n'emporte l'adhésion? comment construire

43. Cf. Bollack 1997, 9 et 12.

44. Cf. Brunschwig 1992, 8o sq. 
la syntaxe d'une expression? à quel mot faut-il rattacher un autre mot comme au verbe dont il est l'objet? comment concevoir le plan d'une série d'arguments? Dans tous les cas, nous n'avons pas choisi de nous appesantir sur un aspect plutôt que sur un autre. Ce sont plutôt les difficultés qui se sont imposées à notre lecture, l'empêchant de passer outre, la retenant dans la difficulté.

On s'installe par là dans ce que le texte a de difficile et d'obscur, en refusant sciemment toute interprétation qui se répandait sur le bien-connu. L'effort entêté pour établir le sens dans la plus grande clarté possible, mais sans exclure d'emblée que le texte puisse être allusif, condensé, ou simplement profond, mène au contraire à manifester que le bien-connu était mal connu. Il nous arrive par conséquent de prendre le contre-pied d'une interprétation parfois ancienne, pour courir le risque d'une restitution de ce que nous croyons être le sens originel. Le dialogue, parfois critique, avec les interprètes du passé, ne mène pas à la prétention ridicule de se croire supérieur à eux. Au contraire, le respect jaillit naturellement de la prise de conscience des difficultés auxquelles ils ont dû faire face ${ }^{45}$.

On le voit, la pratique de la lente lecture, qui arrime solidement la philologie classique à l'herméneutique critique ${ }^{46}$, en fait en quelque sorte un pont reliant la philosophie à l'Altertumswissenschaft et aux sciences historiques en général; car les historiens ont reconnu depuis longtemps l'utilité des études philologiques pour leur propre discipline, puisque certains d'entre eux leur accordent le titre de «sciences auxiliaires traditionnelles ${ }^{47}$.

Une question secondaire subsiste cependant: pourquoi ne pas apprendre la lente lecture en étudiant une autre philologie que la philologie classique? D’abord, parce que c'est la plus ancienne et la plus répandue (la plus universelle) de toutes les philologies particulières, malgré les progrès extraordinaires des philologies germanique et, surtout, romane ${ }^{48}$; puis, parce que le développement des langues et de la culture

\footnotetext{
45. Cf. Brague 1982, $167 s q$.

46. Cf. Bollack 200o, 20 sq.

47. Cf. Samaran 1986, 447-767.

48. Faut-il rappeler ici ce qu'écrivait à ce propos Joseph Bédier, il y a seulement un siècle (Bédier 1903, VII) : «Il existe une méthode déjà presque séculaire, qui fonde la restauration des textes sur le classement critique et sur l'emploi raisonné de toute la tradition manuscrite et imprimée; tandis qu'on l'applique journellement aux œuvres grecques, latines et médiévales, c'est grand'pitié que tant d'œuvres de nos classiques [...] demeurent abandonnées à l'empirisme des éditeurs et à leur caprice»? Un siècle avant lui, l'helléniste Jean-François Boissonade avait cependant préconisé lui aussi l'application aux textes modernes des méthodes ecdotiques jusqu'alors réservées à ceux de l'Antiquité classique (Colincamp 1863, 312): «Dans ces passages douteux, il faudrait une note; il faudrait que l'éditeur rapportât les variantes, les discutât avec un peu de critique, et montrât les raisons qu'il a eues de prendre une leçon de préférence à l'autre. On devrait, ce me semble, appliquer un peu plus souvent aux éditions des livres modernes la méthode que suivent les philologues, quand ils réimpriment les ouvrages de l'antiquité». Mais Boissonade ne fut pas entendu (cf. Bernhard 1910,
} 
classique peut être suivie sur trois ou quatre millénaires, des premières inscriptions en linéaire B jusqu'à nos jours; ensuite, parce que les témoignages écrits sur lesquels repose son étude appartiennent à un très grand nombre de genres littéraires et reflètent une très grande diversité de réalités historiques et d'idées scientifiques, philosophiques, politiques ou religieuses; enfin, parce qu'ils nous sont parvenus sur un grand nombre de supports différents (pierre, coquille d'huître, écorce de bouleau, papyrus, parchemin, volumen, codex, etc.), au terme d'une tradition souvent mouvementée: œuvres complètes, œuvres choisies, anthologies, abrégés, citations, fragments, etc. Le domaine de la philologie classique est donc si riche et si complexe que nul ne peut aujourd'hui en avoir exploré toutes les régions et maîtriser toutes les techniques particulières mises au point durant sa longue histoire.

Mais je reviens à l'inventeur de la brillante correction du texte d'André Chénier. Le philologue Henri Weil ne fut pas seulement un maître en l'art de la critique conjecturale, ce fut assurément aussi un adepte de la lente lecture, car il se tint toujours loin de la "précipitation indécente et suante qui veut tout de suite en avoir fini avec tout, sans excepter l'ensemble des livres anciens et modernes». Selon lui, le sens des chefs-d'œuvre littéraires du passé n'était pas donné, d'ores et déjà connu et reconnu:

Il est des œuvres d'art puissantes et profondes qui s'imposent à l'admiration par la grandeur de la conception, et qui ont en même temps l'attrait de je ne sais quel mystère qui les entoure. Elles font rêver, elles donnent lieu aux interprétations les plus diverses, chacun les entend à sa façon, mais tout le monde s'accorde sur un point, l'admiration du génie qui les a créées. Tel est l'Hamlet de Shakspeare, tel est aussi le Prométhée d'Eschyle ${ }^{49}$.

Il n'est ainsi nullement surprenant que durant plus d'un demi-siècle (1844-1909), il n'ait jamais cessé de s'escrimer à donner une interprétation du tout comme du détail de cette dernière tragédie, à laquelle il travailla régulièrement durant la soixantaine d'années que dura sa carrière de philologue ${ }^{50}$ et à laquelle il travaillait encore, alors

VII: «Aber keinem der Kritiker fiel es ein, sich bei der Untersuchung der philologischen Methode zu bedienen und auf die handschriftliche Überlieferung zurückzugehen»), contrairement à son illustre successeur (Grat 1933, 3) : «Depuis une dizaine d'années, des recherches de savants, au premier rang desquels il faut citer Dom Henri Quentin et M. Joseph Bédier, ont attiré l'attention sur la question de l'édition des textes. Le public érudit s'est rendu compte qu'avant de livrer à l'impression le texte de la plus modeste chronique il fallait faire de nombreuses recherches et résoudre beaucoup de problèmes, et les éditeurs eux-mêmes qui, trop souvent, se contentaient de quelques investigations superficielles, ont été secoués de leur torpeur».

49. Cf. Weil 1886, 280.

50. En témoignent sa thèse sur les allusions aux événements politiques dans les tragédies grecques (1844), sa monographie sur Eschyle et les origines de la tragédie grecque (1849), la «Praefatio» et 
qu'il était devenu complètement aveugle, un mois avant sa mort ${ }^{51}$. Contrairement à l'affirmation de Jean Bollack, suivant laquelle «la critique verbale» avait acquis chez Weil «une autonomie préjudiciable» à la compréhension véritable des textes antiques, «l'intérêt herméneutique pour l'œuvre dans sa nature de production historique ${ }^{52}$ n'était nullement absent de son travail philologique, qui reposait bien entendu sur l'union intime de la critique et de l'herméneutique:

La critique des textes regarde les éditeurs. Mais les lecteurs doivent aussi s'y intéresser jusqu'à un certain point: car il est de leur intérêt qu'elle soit bien faite [...].

Il est temps de terminer cet article, dans lequel j'ai voulu montrer que la critique des textes a ses méthodes, et qu'appliquées avec rigueur et discernement elles peuvent conduire à des résultats nouveaux et certains ou voisins de la certitude. On aura compris encore que, si la critique ne peut être séparée de l'interprétation, celle-ci ne saurait non plus se passer de la critique, et que pour bien expliquer les auteurs anciens, il faut s'enquérir de la constitution de leur texte ${ }^{53}$.

Le regretté Pierre Vidal-Naquet avait naguère beau jeu de moquer des philologues qui, comme Jean Bollack, consacrent «la moitié d'une vie à éditer un texte»

les notes de la première édition qu'il donna du Prométhée enchaîné (1864), ses «Notes sur divers auteurs» (1877), ses "Questions relatives aux nouveaux fragments d'Euripide et d'autres poètes grecs» (1880) ou les recensions qu'il rédigea des Prolégomènes à Eschyle de Rudolf Westphal (1869), des études sur le même poète de Nicklaus Wecklein (1872), des recherches philosophiques de Victor Courdaveaux sur Eschyle, Xénophon et Virgile (1872), de l'édition du texte procurée par Ludwig Schmidt (1874), de la Prométhéide de Théodore-Henri Martin (1876) et des recherches sur les voyages d'Io de Peter Wilhelm Forchhammer (1881); la préface et l'apparat critique de sa seconde édition de la pièce (1884), ses «Remarques sur Eschyle» (1884), une «Notice sur Eschyle» (1884) placée en tête de plusieurs publications scolaires, l'article d'ensemble intitulé «La fable de Prométhée dans Eschyle» (1886), son examen des «traces de remaniements dans les tragédies d'Eschyle» (1888), les comptes rendus qu'il a publiés de l'Introduction à la tragédie attique d'Ulrich von Wilamowitz-Moellendorff (1890), de l'édition du drame par Wecklein (1893) et d'une étude de Paulus Richter sur la dramaturgie d'Eschyle (1894); ou encore les deux révisions qu'il donna de sa seconde édition du Prométhée d'Eschyle (1907 et 1910); pour tout cela, cf. Glotz \& Reinach 1909.

51. Cf. Weil 1910, II: "Parisiis, mense octobri a. MCMIX».

52. Cf. Bollack 1997, 68: «La situation du commentaire français, peu avant Wilamowitz, offre le tableau suivant: d'un côté le double jeu des notes critiques et explicatives qui, chez Tournier, ne s'interpénètrent pas (un peu plus chez Weil), si bien que l'unité de l'objet manque; de l'autre, chez Pierron, la somme des exégèses antérieures depuis les grammairiens alexandrins (auxquels, à travers Lehrs, Pierron se rattache). En apparence, le premier type est plus ouvert, Weil et Tournier cherchant à promouvoir une production nationale de conjectures, mais la problématique est atomisée (et interchangeable), et la critique verbale prend une autonomie préjudiciable; le second type, plus préoccupé d'informer des lecteurs que l'on devine privés de bibliothèques, est fermé sur la totalité qu'il offre. Dans les deux cas, l'intérêt herméneutique pour l'œuvre dans sa nature de production historique semble absent».

53. Cf. Weil 1866, 162;165. 
et qui, comme un consciencieux «érudit portugais» de sa connaissance, veulent avoir tout lu de la bibliographie de leur sujet:

J'ai très vite compris les limites de l'érudition pure. Je pense à un érudit portugais qui a fait une thèse sur Platon. Il a tout lu sur Socrate et sur Platon, absolument tout, et il n'en tire finalement aucune conclusion ${ }^{54}$.

Il commettait cependant une petite erreur : il ne s'agit nullement là d'érudition, mais de la saine pratique philologique de la lente lecture, qui me parait être la seule méthode connue pour parvenir à la compréhension d'un texte. Si l'érudit portugais n'est parvenu à aucun résultat après ses longues lectures, il n'en était pas moins nécessaire qu'il les menât à bien pour savoir qu'elles ne menaient à rien.

\section{Une discipline maîtresse de la formation universitaire}

On a vu plus haut la critique adressée par Bœckh à la célèbre définition de l'Altertumswissenschaft formulée par Friedrich-August Wolf en 1807. Celle-ci ne souffrait toutefois pas seulement d'un excès d'empirisme, car elle laissait dans l'ombre la question cruciale de la nécessité d'une telle science. La chose n'a pas échappé à Max Bonnet (1845-1917), qui remarquait dans sa leçon sur l' «Histoire de la Philologie», prononcée à l'université de Montpellier au mois de décembre 1890:

Il ne manque qu'une chose à cette définition, c'est de dire plus exactement pourquoi il nous faut cette vive intelligence des œuvres classiques, fondée sur cette exacte connaissance du monde ancien ${ }^{55}$.

La réponse qu'il apportait lui-même à cette question - et qui était d'ailleurs largement conforme aux conceptions de Wolf - satisfaisait sans doute aux aspirations des lecteurs cultivés de son époque:

Il nous la faut, non seulement afin de nous approprier à nous-mêmes la substance et l'esprit des œuvres anciennes, mais particulièrement afin de pouvoir en tirer le fruit qu'on en attend pour l'éducation de la jeunesse. Et c'est bien ainsi que depuis Wolf on l'entend dans la pratique, si on ne le reconnait pas assez en théorie. C'est sur ce fait que repose depuis cette époque toute l'organisation des études philologiques en Allemagne et dans la plupart des autres pays, chez nous-mêmes aussi, depuis que des études régulières en vue de la carrière de l'enseignement ont été organisées dans nos facultés ${ }^{56}$.

54. Cf. Vidal-Naquet 2006, 64 sq.

55. Cf. Bonnet 1892, 66 sq.

56. Ibid., 67 . 
Dans le contexte socioculturel et scientifique qui est le nôtre, je ne suis toutefois pas bien certain que «l'éducation de la jeunesse» ne se puisse pas mener à bien sans appropriation de «la substance» et de «l'esprit des œuvres anciennes». Dans notre société mondialisée, on ne peut plus en effet affirmer, avec Johann Wolfgang Goethe (1749-1832), que «les antiquités chinoises, indiennes, égyptiennes ne sont jamais que des curiosités » et «auront peu d'effet sur notre formation morale et esthétique " ${ }^{57}$, sans être immédiatement contredit par la réalité objective des agences de voyages, des expositions de musées et des présentoirs de librairies; ni soutenir, avec Salomon Reinach (1858-1932), que «la littérature, la philosophie et l'art» de l'Antiquité classique "sont les sources toujours vives de la culture moderne» ${ }^{58}$, sans exclure du même coup du champ de la culture contemporaine l'immense masse de tout ce qui ne se ressent pas de l'influence gréco-romaine. Les professeurs de langues et de littératures anciennes rougiraient plutôt, je pense, de reprendre aujourd'hui à leur compte les propos que tenait jadis Émile Egger (1813-1885), dans un morceau intitulé «D’une renaissance nouvelle des études grecques et latines au dix-neuvième siècle» (1869):

Mais si la Grèce et Rome perdent, au profit de l'humanité, le privilège d'attirer seules notre attention, elles en gardent d'impérissables par la supériorité de leur génie, par les rapports étroits que l'affinité naturelle et l'éducation maintiennent entre ces deux grands peuples et les peuples chargés par la Providence de diriger aujourd'hui le progrès de la civilisation ${ }^{59}$.

Je nourris certes une profonde admiration pour les travaux scientifiques d'un savant qui joua un rôle de toute première importance dans la renaissance des études grecques en France au XIX ${ }^{e}$ siècle ${ }^{60}$; mais en relisant ces lignes, je ne puis m'empêcher de songer que le grand helléniste, qui fut un familier des "Conférences populaires faites à l'Asile impérial de Vincennes sous le patronage de S.M. l'Impératrice», ne sut pas toujours se tenir à l'écart du pouvoir impérial, lequel n'avait assurément pas été chargé "par la Providence», mais s'était lui-même chargé par un coup d'État, de diriger alors «le progrès de la civilisation», c'est-à-dire de promouvoir le développement du capitalisme industriel et financier, dont la crise actuelle, après Jules Vallès (1832-1885) et Émile Zola (1840-1902), ne manque pas de remettre en cause le bien-fondé ${ }^{61}$. L'une desdites "Conférences populaires", intitulée "Les projets de réforme sociale dans l'Antiquité» (1867) et prononcée trois ans à peine après

57. Cf. Goethe $1833,126$.

58. Cf. Reinach $1883,3$.

59. Cf. Egger 1869, II, 440.

60. Outre l'ouvrage mentionné ci-dessus, je pense en particulier à : Egger 1849, Egger 1854, Egger 1862, Egger 1863 et Egger 1866.

61. Cf. Bailly 1886, 74-82; cet aspect du personnage est en revanche complètement gommé dans Wallon 1893. 
la formation à Londres de l'Association internationale des travailleurs, avait ainsi pour but avoué de défendre les prolétaires convalescents auxquels elle était adressée "contre la séduction des théories dangereuses» en tirant de l'histoire grecque «un enseignement agréable, autant qu'instructif», sur «ces funestes systèmes » que sont les «utopies égalitaires et communistes ${ }^{62}$. Et le savant professeur d'entrer bien entendu en matière par l'évocation des liens héréditaires et disciplinaires qui nous unissent à la Grèce ancienne:

Nous sommes, à bien des égards, les héritiers et les élèves du peuple grec dans les sciences, dans les lettres, dans les beaux-arts; il n'a guère eu de maîtres, et il a été lui-même le maître de presque tous les peuples de l'Occident; en politique, il a le premier donné l'exemple des institutions dont nous nous efforçons de développer les principes; il a le premier essayé d'organiser la souveraineté populaire, et, quoiqu'il n'ait qu'à moitié réussi dans cette tâche difficile, il a droit à tous nos respects pour ses généreux efforts, à toute notre attention pour les œuvres qu'a produites son ingénieuse et féconde activité ${ }^{63}$.

Puis de louer Aristophane d'avoir cherché, par les traits piquants de la satire, à détourner ses concitoyens de leur manie "pour les nouveautés politiques et sociales" et de leur avoir montré l'inutilité de "porter remède par la violence» à «l'inégale répartition des richesses » ${ }^{64}$; de féliciter l'ingénieux Aristote d'avoir réfuté «le système d'un certain Phaléas, qui, comme ces femmes athéniennes dans la comédie d'Aristophane [...], prétendait établir l'égalité de biens entre les citoyens d'une même république » ${ }^{65}$; et de mentionner au passage les peuples colonisés, «qui nous doivent leur civilisation", la grande religion catholique, "si supérieure à celle des Grecs», et les «devoirs à remplir» de chacun ${ }^{66}$, etc.

À ce compte et avec un tel humanisme, on aurait aussi pu arguer de certaines satires de Juvénal, qui sont pour le moins misogynes, homophobes et xénophobes, pour priver les femmes du droit de vote, interdire à la Grèce l'entrée dans la Communauté européenne et fermer à jamais les frontières de notre pays à toute immigration. La culture française est certes fille de l'Antiquité classique, mais elle a quand même fini par s'en émanciper. On peut le regretter, mais c'est ainsi. D'ailleurs, la Grèce elle-même avait bien dû s'affranchir elle aussi des liens dans lesquels la tenait tout d'abord la culture indo-européenne et s'était laissé influencer par les Égyptiens, les Phéniciens et d'autres peuples, sans doute, de la Méditerranée orientale.

\footnotetext{
62. Cf. Egger 1867,$8 ; 42 ; 49$.

63. Ibid., 8 sq.

64. Ibid., 16 sq.; 23.

65. Ibid., 33 .

66. Ibid., 43 et 49 .
} 
Mais je reviens à la question cruciale de la nécessité de la science de l'Antiquité. Il est incontestable que le monde de l'Antiquité classique est un sujet d'études extrêmement riche et fécond; et que ce qu'on appelle le monde occidental entretient avec lui des relations politiques, scientifiques et culturelles qu'il n'a pas avec l'Afrique, l'Amérique, l'Océanie, la Chine ou l'Inde anciennes. Mais ces relations privilégiées ne justifient pas nécessairement la place prépondérante qu'occupe encore aujourd'hui la science de l'Antiquité au sein des sciences humaines, de l'Université et de tout le système éducatif français, car notre pays entretient également avec l'Afrique, l'Amérique, l'Océanie, la Chine et l'Inde modernes des relations économiques, politiques, scientifiques et culturelles qu'il n'a nullement avec le monde de l'Antiquité classique. Selon moi, cette place est cependant légitime et devrait même être renforcée: ce qui est là en jeu, ce n'est pas la connaissance de l'Antiquité classique en elle-même (son histoire, ses langues, sa littérature, sa philosophie, ses valeurs, sa culture), ce n'est pas la conservation, l'accroissement et la diffusion de toutes ces belles connaissances, mais bien l'éducation même de la jeunesse: la formation et le développement de son intelligence et de sa propre culture, c'est-à-dire de sa faculté de comprendre le monde qui l'entoure et dont elle est partie prenante. Ce qui est ici primordial, ce n'est pas la science théorique de l'Antiquité, c'est la science pratique de la philologie classique, qui en est comme le couronnement ${ }^{67}$. Je ne vois, en effet, pas bien ce qui, dans l'enseignement supérieur des lettres, pourrait se substituer à l'apprentissage de la lente lecture: certainement pas, en tout cas, la linguistique structurale, pragmatique ou générative, l'analyse génétique, psychocritique ou sociocritique des textes, ni l'histoire générale de la pensée, de l'art ou de la littérature, qui ne sauraient en être que des auxiliaires. Je ne vois pas non plus ce qui, dans l'enseignement secondaire, pourrait remplacer l'initiation à la lecture philologique, telle que certains enseignants courageux la pratiquent parfois encore en confrontant directement les élèves aux problèmes posés par les textes antiques dans leur langue originale, quand cette initiation n'a pas laissé sa place à un prétendu apprentissage ludique, fondé sur une admiration béate, des jeux infantiles, des tapotages cybernétiques et des documents audiovisuels: certainement pas, en tout cas, la grammaire des textes et la lecture dite méthodique, qui font abstraction du caractère historique, esthétique et problématique des chefs-d'œuvre du passé et se satisfont d'une compréhension globale du déjà connu.

L'on peut certes pointer du doigt le caractère sclérosant de la philologie classique du passé, telle qu'elle fut pratiquée et enseignée comme «discipline maîtresse de la formation universitaire jusqu'au début» du $\mathrm{XX}^{\mathrm{e}}$ siècle ${ }^{68}$. Mais il convient alors

67. Cf. Bonnet 1892, 1-36.

68. Cf. Bollack 1997, 29. 
d'entonner la protestation de Frédéric Nietzsche $(1871)^{69}:$ :On croit que c'est la fin de la philologie, je crois qu'elle n'a pas encore commencé»; et de soumettre les méthodes philologiques aux impératifs d'une nouvelle science, l'herméneutique critique $^{70}$, qui oriente un admirable rapport sur une mission ministérielle relative à l'avenir des études classiques en France (2004). On y peut lire ceci:

Devant un texte grec ou latin, le lecteur ne peut simplement appliquer des règles. Il ne dispose pas, comme pour les langues vivantes actuelles, du code qui a encadré l'expression de l'auteur [...]. Comme il n'y a pas de langue en dehors des textes, les règles de la langue doivent être reconstruites à partir de la lecture, elles sont élaborées comme des hypothèses à partir des phrases et de leur enchaînement. [...] Le lecteur, l'élève, est ainsi mis dans une situation expérimentale où il sait qu'il y a bien un sens à découvrir, mais où il doit, pour y arriver, s'en donner les moyens. Cela vaut aussi pour les mots qu'il aura à traduire. Ces mots entrent dans des systèmes sémantiques, propres à une époque, à un auteur et parfois à un texte. Leur sens ne pourra pas être tiré directement du dictionnaire: il faudra, chaque fois, vérifier si le sens qu'un dictionnaire lui propose est adéquat à tel emploi particulier, et le lecteur sera amené pour cela à un travail de comparaison avec d'autres emplois. [...]

La lecture ne pourra aboutir, c'est-à-dire produire du sens, que si le même travail est accompli au niveau du contenu historique de l'expression. Les mots et les phrases d'un texte ne sont pas seulement les éléments d'un jeu formel, mais renvoient à un contexte historique donné, qu'ils expriment, et transforment ou visent à transformer par la manière dont ils le représentent [...].

Le texte antique, si l'élève passe par ce moment méthodique de la construction, devient sien; il peut alors le comparer aux textes plus modernes qui le traduisent, l'imitent, le réécrivent; il peut aussi le comparer à ce qu'il écrirait lui-même dans sa propre langue. Ce n'est ainsi pas seulement un texte qu'il se réapproprie, mais une tradition. [...]

Cet apprentissage est de portée générale. Il met dans une situation exemplaire et irremplaçable pour tout rapport à la culture, quelle qu'elle soit, proche ou lointaine ${ }^{71}$.

Les autorités politiques ne paraissent pas avoir pris connaissance de ce rapport ni conscience de son importance: son objet n'est pourtant autre que l'avenir de la culture et de l'esprit de finesse dans le pays de Blaise Pascal (1623-1662) ${ }^{72}$.

69. Cf. Nietzsche 1992, 55 .

70. Cf. Bollack 2000, 79-157.

71. Cf. Judet de la Combe \& Wismann 2004, 221-225.

72. Cf. Bonnet 1892, 28 sq.: «Ce n'est pas tout que l'esprit géométrique; il faut encore, pour faire un bon étudiant, de quelque Faculté que ce soit, l'esprit de finesse. Ce n'est pas assez, même pour étudier la chimie ou la physique, ni à plus forte raison la médecine, le droit ou l'histoire, de posséder 


\section{Conclusion}

Le moment est venu de la récapitulation et de la formulation de la thèse que défend la présente communication. Les sciences de l'Antiquité ont certes leur intérêt propre, qui n'est pas mince, étant donné la place occupée par la culture classique dans l'histoire du monde; elles en présentent cependant un autre, qui est indirect mais a une valeur plus universelle encore: elles procurent à la philologie classique, qui est une science pratique à visée pédagogique, les matériaux et les méthodes permettant aux philologues de former des professeurs des écoles et des enseignants de lettres dignes de ce nom; et à ces derniers de prodiguer l'enseignement de la lecture intelligente dans les lycées, les collèges et les écoles primaires.

Attenter au développement des sciences de l'Antiquité et de la philologie classique dans l'Université française, c'est donc tout simplement saper les fondements du

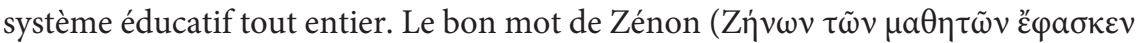

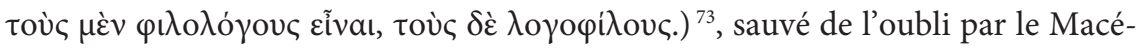
donien Stobée, aura en tout cas perdu tout son sel, quand, parmi les élèves et les étudiants, appelés à devenir les enseignants de l'avenir, il n'y aura plus que de beaux

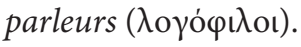

Laurent CALvié

CPAF-UMR 6125,

Université de Provence - Aix-Marseille I

les notions qu'on peut donner à de jeunes élèves sur les choses de la nature, toutes relativement simples, planes, rectilignes; il faut encore avoir quelque habitude des choses de l'esprit, qui sont infiniment plus complexes, pleines de replis et ondoyantes, dont l'étude exige par conséquent des méthodes et des procédés plus variés et plus délicats».

73. Cf. Stob. III, 36, 26, que je traduirais ainsi : «Zénon dit qu'il y a deux sortes d'élèves : les philologues et les logophiles». 


\section{Références bibliographiques}

\section{Auteurs anciens}

Denys le Thrace (Lallot 1989), La Grammaire, J. Lallot (éd. et trad.), Paris, CNRS (Sciences du langage).

Dionysii Thracis Ars grammatica, G. Uhlig (éd.), Leipzig, B.G. Teubner (Grammatici Graeci; I I), 1883 (réimpression anastatique par Georg Olms Verlagsbuchhandlung, Hildesheim, 1965).

Scholia in Dionysii Thracis Artem grammaticam, A. Hilgard (éd.), Leipzig, B.G. Teubner (Grammatici Graeci; I III), 1901 (réimpression anastatique par Georg Olms Verlagsbuchhandlung, Hildesheim, 1965).

StobéE (Hense 1894), Anthologii libri duo posteriores, O. Hense (éd.), Berlin, Weidmann, t. I.

C. Suetoni Tranquilli De grammaticis et rhetoribus, R.-P. Robinson (éd.), Paris, Librairie ancienne Édouard Champion, 1925.

\section{Études}

Bailly A. (1886), Notice sur Émile Egger, Orléans, Imprimerie de Georges Jacob.

Baratin M., Desbordes F. (1981), L'analyse linguistique dans l'Antiquité classique. 1. Les théories, Paris, Klincksieck (Horizons du langage).

BÉDIER J. (1903), Études critiques, Paris, Armand Colin, 1903.

Benoist E. (1877), «Frédéric Ritschl», RPh, 1, p. 91-10o.

Bernhard A. (1910), Die Parodie “Chapelain décoiffé, Leipzig, A. Deichert'sche Verlagsbuchhandlung Nachf. (Münchener Beiträge zur Romanischen und Englischen Philologie; 50).

Вєескн А. (1877), Encyklopädie und Methodenlehre der philologischen Wissenschaften, Leipzig, B.G. Teubner.

Вєскн А. (1991), La Filologia come scienza storica. Enciclopedia e metodologia delle scienze filologiche, R. Masullo (trad.), Naples, Guida editori (Micromegas) ( $1^{\text {re }}$ édition: 1987).

BolLACK J. (1996), «La référence allemande dans les études philologiques à l'École normale», in L'École normale supérieure et l'Allemagne, M. Espagne (éd.), Leipzig, Leipziger Universitätsverlag (Deutsch-französische Kulturbibliothek; 6), p. 23-38.

Bollack J. (1997), La Grèce de Personne. Les mots sous le mythe, Paris, Seuil (L'ordre philosophique).

Bollack J. (2000), Sens contre sens. Comment lit-on? Entretiens avec Parick Llored, Genouilleux, La passe du vent. 
Bonnet M. (1892), La philologie classique. Six conférences sur l'objet et la méthode des études supérieures relatives à l'Antiquité grecque et romaine, Paris, Librairie Klincksieck.

Brague R. (1982), Du temps chez Platon et Aristote. Quatre études, Paris, PUF (Épiméthée [= Quadrige; 409]).

Brambach W. (1865), Friedrich Ritschl und die Philologie in Bonn, Leipzig, B.G. Teubner.

BRUNSCHWIG J. (1992), «Faire de l'histoire de la philosophie aujourd'hui », in Nos Grecs et leurs modernes. Les stratégies contemporaines d'appropriation de l'Antiquité, B. Cassin (éd.), Paris, Seuil (Chemins de pensée), p. 67-96 (reproduit le texte d'une « conférence [...] faite en 1976 devant la Société française de philosophie» et publiée dans le Bulletin de la Société française de philosophie, 71 / 4, 1976, p. 125-149).

Carcopino J. (1968), Les bonnes leçons, Paris, PUF (À la pensée).

Chénier A. (1819), Euvres complètes, Paris, Baudoin frères, Foulon et $\mathrm{C}^{\mathrm{ie}}$.

Chénier A. (1826), Euvres posthumes, Paris, Guillaume.

ChÉnier A. (1832), Poésies posthumes et inédites, Paris, Charpentier et Eugène Renduel.

Chénier A. (1852), Poésies, Paris, Charpentier.

Chénier A. (1874), Euvres poétiques, Paris, A. Lemerre.

ChÉnier A. (1958), Euvres complètes, Paris, Gallimard (Pléiade; 57).

Colincamp F. (1863), J.-F. Boissonade, critique littéraire sous le premier Empire, Paris, Librairie académique Didier et $\mathrm{C}^{\mathrm{ie}}$, t. II.

Dahlmann H. (1970), Zur Ars grammatica des Marius Victorinus, Mayence - Wiesbaden, Franz Steiner Verlag (Abhandlungen der Geistes- und Sozialwissenschaftlichen Klasse ; 2$)$.

EgGer É. (1849), Essai sur l'histoire de la critique chez les Grecs. Introduction à l'étude de la littérature grecque, Paris, A. Durand ( 2 édition, revue, corrigée et augmentée: Paris, G. Pendone-Lauriel, 1886).

EgGer É. (1854), Apollonius Dyscole. Essai sur l'histoire des théories grammaticales dans l'Antiquité, Paris, A. Durand (réimpr.: Hildesheim - New York, G. Olms (Documenta Semiotica, série 1), 1987).

EgGer É. (1862), Mémoires de littérature ancienne, Paris, A. Durand.

EgGer É. (1863), Mémoires d'histoire ancienne et de philologie, Paris, A. Durand.

EGGER É. (1866), Études historiques sur les traités publics chez les Grecs et chez les Romains, depuis les temps les plus anciens jusqu'aux premiers siècles de l'ère chrétienne, Paris, A. Durand.

EgGer É. (1867), Études d'histoire ancienne. Les projets de réforme sociale dans l'Antiquité, Paris, Hachette (Conférences faites à l'Asile impérial de Vincennes, sous le patronage de S.M. l'Impératrice). 
EgGer É. (1869), L'Hellénisme en France. Leçons sur l'influence des études grecques en France dans le développement de la langue et de la littérature françaises, Paris, Didier, 2 vol.

Flamand J.-M. (1994), "Cratès de Mallos», in Dictionnaire des philosophes antiques. II. Babélyca d'Argos à Dyscolius, R. Goulet (éd.), Paris, CNRS, p. 487-495.

France A. (1924), Le lys rouge, Paris, Calmann-Lévy.

Glotz G., Reinach T. (1909), «Bibliographie scientifique de Henri Weil (1818-1909)», $R E G, 22 / 100$, p. 383-404.

Goethe J.W. (1833), Nachgelassene Werke, Stuttgart - Tübingen, J.G. Cotta, t. IX.

Grat F. (1933), «L'histoire des textes et les éditions critiques», Bibliothèque de l'École des chartes, 94, p. 3-16.

Hemmerdinger B. (1972), «Henri Weil. Entretien avec Madame Dufour», Belfagor, 27, p. 96-99.

Judet de la Combe P., Wismann H. (2004), L'avenir des langues. Repenser les Humanités, Paris, Éditions du Cerf (Passages).

Nietzsche F. (1954), Werke in drei Bänden, K. Schlechta (éd.), Munich - Vienne, Carl Hanser Verlag, t. I.

Nietzsche F. (1989), Aurore. Pensées sur les préjugés moraux, Paris, Gallimard (FolioEssais; 119).

Nietzsche F. (1992), Sur la personnalité d'Homère, suivi de Nous autres Philologues, Nantes, Le Passeur - Cecofop.

Perrot G. (1910), Notice sur la vie et les travaux de Henri Weil, Paris, Firmin-Didot et $C^{\text {ie }}$.

Peyre H. (1932), Bibliographie critique de l'Hellénisme en France, New Haven, Yale University Press (Yale Romanic Studies; 6).

ReInACH S. (1883), Manuel de philologie classique, Paris, Librairie Hachette et $\mathrm{C}^{\mathrm{ie}}$, t. I ( $2^{\mathrm{e}}$ édition revue et augmentée).

ReInach T. (1909), «Discours aux obsèques de M. Henri Weil», REG, 22/100, p. 379-382.

Ritschl F. (1879), Kleine philologische Schriften. Band V. Vermischtes, Leipzig, B.G. Teubner.

Samaran C. (éd.) (1986), L'Histoire et ses méthodes, Paris, Gallimard (Encyclopédie de la Pléiade; 11).

Simon-Nahum P. (2003), "La lettre et le sens», Critique, 672, p. 332-345.

VAlors H. de (1740), Henrici Valesii Emendationum libri quinque et De critica libri duo, Amstelaedami, Apud Salomonem Schouten.

VIDAL-Naquet P. (2006), L'Histoire est mon combat. Entretiens avec Dominique Bourel, Paris, Albin Michel (Itinéraires du savoir). 
Wallon H. (1893), "Notice sur les travaux de M. Émile Egger», Mémoires de l'Académie des Inscriptions et Belles-Lettres, 35/1, p. 345-368.

WeIL H. (1838), Ueber Thukydides als Geschichtsschreiber, Darmstadt, C.W. Leske.

WeIL H. (1844 a), De tragœdiarum græearum cum rebus publicis conjunctione, Paris, Crapelet.

WeIL H. (1844 b), De l'Ordre des mots dans les langues anciennes comparées aux langues modernes. Question de grammaire générale, Paris, Joubert ( $2^{\mathrm{e}}$ édition: FranckVieweg, 1869; $3^{\mathrm{e}}$ édition: Vieweg, 1879).

Weil H. (1847), «Des tragédies perdues d’Euripide. - I. Antiope », Journal général de l'instruction publique, p. 850-853; 858-861.

Weil H. (1859), «Restitution d'un chœur d'Eschyle», Journal général de l'instruction publique, p. 397-399.

Weil H. (1861 a), «Ch. Thurot, Études sur Aristote, Politique, Dialectique, Rhétorique», Journal général de l'instruction publique, p. 278-280 [p. 279: Restitution du vers d'André Chénier].

WeIL H. (1861 b), «La loi de symétrie appliquée à l'explication d'un passage lyrique des Perses d'Eschyle», Journal général de l'instruction publique, 15, p. 112-113.

WeIL H. (1866), «De la méthode à suivre pour restituer les textes anciens », Revue de l'instruction publique, p. 162-165.

Weil H. (1886), "La fable de Prométhée dans Eschyle», Annuaire de l'Association pour l'encouragement des études grecques en France, 20, p. 280-299.

WEIL H. (1890), compte rendu d' «EURIPIDES HERAKLES, erklärt von Ulrich von Wilamowitz-Moellendorff. - Volume I. Einleitung in die Attische Tragödie. - Volume II. Text und Commentar, Berlin, Weidmann, 1888 ", Journal des savants, p. 201-219.

Weil H. (1910), Aeschyli Tragoediae, Lipsiae, In aedibus B.G. Teubneri (BT) (2 édition revue [ $1^{\text {re }}$ éd. : $1884 ; 2^{\mathrm{e}}$ éd. : 1907$\left.]\right)$.

WeIl H. (2010), Études sur l'ancienne poésie grecque, Toulouse, Anacharsis (Essais), sous presse.

Weil H., Benloew L. (1855), Théorie générale de l'accentuation latine, suivie de recherches sur les inscriptions accentées, et d'un examen des vues de M. Bopp sur l'histoire de l'accent, Paris - Berlin, A. Durand et F. Dümmler.

Wilamowitz-Moellendorf U. von (1921), Einleitung in die griechische Tragödie, Berlin, Weidmannsche Buchhandlung.

Wolf F.A. (1807), «Darstellung der Alterthums-Wissenschaft nach Begriff, Umfang, Zweck und Wert», in Museum der Altertums-Wissenschaft, F.A. Wolf, P. Buttmann (éd.), vol. 1, Berlin, Realschulbuchhandlung. 